\title{
Topological Mass Generation in Four Dimensions
}

\author{
Gia Dvali ${ }^{a}$, R. Jackiw ${ }^{b}$ and So - Young $\mathbf{P i}^{c}$ \\ ${ }^{a}$ Department of Physics and Center for Cosmology and Particle Physics, New York University \\ New York, NY 10003, USA \\ ${ }^{b}$ Center for Theoretical Physics, Massachusetts Institute of Technology \\ Cambridge, MA 02139, USA \\ ${ }^{c}$ Department of Physics, Boston University \\ Boston, MA 02215, USA \\ MIT-CTP/3704 \\ BUHEP-05-19
}

\begin{abstract}
Schwinger's mechanism for mass generation relies on topological structures of a 2dimensional gauge theory. In the same manner, corresponding 4-dimensional topological entities give rise to topological mass generation in four dimensions.
\end{abstract}




\section{Introduction}

In this paper we show that presenting the bosonic sector of the Schwinger model [1] (massless QED in 2-dimensional space-time) by variables dual to the usual ones results in a formulation of the model in terms of well known topological entities: Chern-Pontryagin density $\mathcal{P}$ and Chern-Simons current $\mathcal{C}^{\alpha}, \partial_{\alpha} \mathcal{C}^{\alpha}=\mathcal{P}$. Thus the mass generation in the Schwinger model can be described in topological terms. Moreover, the same topological structures [2], when elevated to 4-dimensional space-time, provide a partial, 4-dimensional generalization of the Schwinger model, together with its mechanism for generating a mass. In Section II, we review the 2dimensional model and emphasize the central role in the mass generation scenario of the chiral anomaly, which is famously related to a topological term. This suggests employing topological entities when describing the model's dynamics. Such a topological formulation is given in Section III, which is then promoted to four dimensions in Section IV. A commentary on our results comprises the last Section V.

\section{Schwinger Model Resumé}

In the Schwinger model, an Abelian vector potential $A_{\mu}$ interacts with a vector current $\mathcal{J}^{\mu}$ constructed from massless Dirac fields $\psi$. The Lagrange density reads

$$
\begin{gathered}
\mathcal{L}=-\frac{1}{4} F^{\mu \nu} F_{\mu \nu}+i \bar{\psi} \gamma_{\mu}\left(\partial_{\mu}+i e A_{\mu}\right) \psi, \quad F_{\mu \nu} \equiv \partial_{\mu} A_{\nu}-\partial_{\nu} A_{\mu} \\
\mathcal{L}_{I}=-e \mathcal{J}^{\mu} A_{\mu}, \quad \mathcal{J}^{\mu}=\bar{\psi} \gamma^{\mu} \psi
\end{gathered}
$$

The traditional solution of the model proceeds by functionally integrating the Dirac fields, giving an effective action.

$$
\mathcal{I}_{e f f}(A)=-\frac{1}{4} \int F^{\mu \nu} F_{\mu \nu}-i \ln \operatorname{det}\left[\gamma^{\mu}\left(\partial_{\mu}+i e A_{\mu}\right)\right]
$$

The functional determinant can be computed because the only non vanishing Feynman diagram is the vacuum polarization graph.

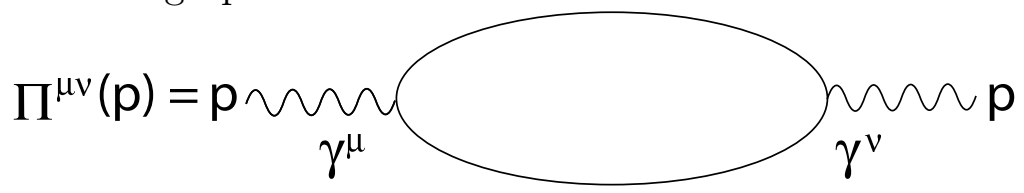

Figure 1: Vacuum polarization graph generates the polarization operator $\Pi^{\mu \nu}(p) \propto\left(g^{\mu \nu}-p^{\mu} p^{\nu} / p^{2}\right)$. This generates the polarization operator $\Pi^{\mu \nu}(p) \propto\left(g^{\mu \nu}-p^{\mu} p^{\nu} / p^{2}\right)$. The coefficient of $g^{\mu \nu}$ 
is evaluation dependent (the diagram is superficially divergent), but it becomes fixed by the gauge invariance requirement that the vector current correlator (whose proper part is $\Pi^{\mu \nu}$ ) be transverse. The effective action

$$
\mathcal{I}_{\text {eff }}(A)=\int\left[-\frac{1}{4} F^{\mu \nu} F_{\mu \nu}+\frac{e^{2}}{2 \pi} A_{\mu}\left(g^{\mu \nu}-\frac{\partial^{\mu} \partial^{\nu}}{\partial^{2}}\right) A_{\nu}\right],
$$

exhibits the generated mass, $m^{2}=\frac{e^{2}}{\pi}$. Although usually one says that the photon acquires a mass, in two dimensions the photon field $A_{\mu}$ can be decomposed as $A_{\mu}=\partial_{\mu} \theta+\epsilon_{\mu \nu} \partial^{\nu} \eta^{\prime}$. The gauge part decouples; only the pseudoscalar $\eta^{\prime}$ remains. So one could just as well say that a pseudoscalar excitation acquires the mass.

It is important to appreciate that the axial vector current $\mathcal{J}_{\alpha}^{5}$, which is conserved with massless fermions within classical dynamics, acquires an anomalous divergence upon quantization. This is immediately seen when the 2-dimensional duality relation between axial and vector currents is used.

$$
\mathcal{J}_{\alpha}^{5}=\epsilon_{\alpha \mu} \mathcal{J}^{\mu}
$$

Formula (2.5) is a consequence of 2-dimensional geometry: when $\mathcal{J}^{\mu}$ is a vector, $\mathcal{J}_{\alpha}^{5}$ defined by (2.5) is an axial vector. More explicitly, (2.5) is seen in a 2-dimensional gamma matrix identity.

$$
\gamma_{\alpha} \gamma^{5}=\epsilon_{\alpha \mu} \gamma^{\mu}
$$

Therefore, the correlator ${ }^{5} \Pi_{\alpha}^{\nu}$ of $\mathcal{J}_{\alpha}^{5}$ with $\mathcal{J}^{\nu}$ can be simply obtained from $\Pi^{\mu \nu}$ as ${ }^{5} \Pi_{\alpha}{ }^{\nu}=$ $\epsilon_{\alpha \mu} \Pi^{\mu \nu}$. Moreover, once a transverse form for $\Pi^{\mu \nu}$ is fixed by gauge invariance, ${ }^{5} \Pi_{\alpha}{ }^{\nu}$ fails to be transverse in the $\alpha$ index; the divergence of the axial vector current is anomalous.

$$
\partial^{\alpha} \mathcal{J}_{\alpha}^{5}=-\frac{e}{2 \pi} \epsilon^{\mu \nu} F_{\mu \nu}=\frac{e}{\pi} F
$$

In the second equality we have introduced the (pseudo) scalar $F$, dual in two dimensions to the anti symmetric $F_{\mu \nu} \equiv \epsilon_{\mu \nu} F$

The anomaly provides an immediate derivation of the mass [3]. We begin with the gauge field equation of motion that follows from (2.1).

$$
\partial_{\mu} F^{\mu \nu}=e \mathcal{J}^{\nu}
$$

In terms of the dual field strength this reads

$$
\epsilon^{\mu \nu} \partial_{\mu} F=e \mathcal{J}^{\nu}
$$

The $\epsilon$ symbol may be transferred to the right side and $\mathcal{J}^{\nu}$ becomes replaced by its dual $\mathcal{J}_{\alpha}^{5}$.

$$
\partial_{\alpha} F=-e \mathcal{J}_{\alpha}^{5}
$$


A further divergence gives the d'Alembertian on the left and the anomaly (2.7) on the right.

$$
\partial^{2} F+\frac{e^{2}}{\pi} F=0
$$

This demonstrates that the pseudoscalar $F$ acquires a mass, $m^{2}=\frac{e^{2}}{\pi}$.

\section{Topological Entities in the Schwinger Model}

The 2-dimensional anomaly is proportional to $-F=\frac{1}{2} \epsilon^{\mu \nu} F_{\mu \nu}$, which is recognized as the 2-dimensional Chern-Pontryagin density $\mathcal{P}_{2}$.

$$
\mathcal{P}_{2}=\frac{1}{2} \epsilon^{\mu \nu} F_{\mu \nu}
$$

Furthermore, the gauge potential $A_{\mu}$ is dual to the Chern-Simons current $\mathcal{C}_{2}^{\alpha}$,

$$
\mathcal{C}_{2}^{\alpha} \equiv \epsilon^{\alpha \mu} A_{\mu}
$$

whose divergence forms the Chern-Pontryagin density [2].

$$
\partial_{\alpha} \mathcal{C}_{2}^{\alpha}=\epsilon^{\alpha \mu} \partial_{\alpha} A_{\mu}=\frac{1}{2} \epsilon^{\alpha \mu} F_{\alpha \mu}=\mathcal{P}_{2}
$$

The bosonic portion of the Lagrange density for the Schwinger model may be written in terms of these topological entities.

$$
\begin{aligned}
\mathcal{L}_{2} & =-\frac{1}{4} F^{\mu \nu} F_{\mu \nu}-e \mathcal{J}^{\mu} A_{\mu}=\frac{1}{2} F^{2}-e A_{\mu} \epsilon^{\mu \alpha} \mathcal{J}_{\alpha}^{5} \\
& =\frac{1}{2} \mathcal{P}_{2}^{2}+e \mathcal{C}_{2}^{\alpha} \mathcal{J}_{\alpha}^{5}
\end{aligned}
$$

Moreover, since $\mathcal{C}_{2}^{\alpha}$ and $A_{\mu}$ are linearly related, it makes no difference which one is the fundamental variable. Thus varying $\mathcal{C}_{2}^{\alpha}$ in (3.4) gives (2.10) directly as the equation of motion.

$$
-\partial_{\alpha} \mathcal{P}_{2}+e \mathcal{J}_{\alpha}^{5}=0
$$

A further divergence and the anomaly equation (2.7) reproduce (2.11), since $\mathcal{P}_{2}=-F$.

It is this last, topological reformulation of the Schwinger model that we shall take to four dimensions. However, we must still address an important point that will arise in the 4-dimensional theory. Observe that the equation of motion (2.10) or (3.5) entails an integrability condition: Since the (axial) vector $\mathcal{J}_{\alpha}^{5}$ is set equal to a gradient of (the pseudoscalar) $\mathcal{P}_{2}$, it must be that the curl of the axial vector vanishes. Equivalently, the dual of the axial vector must be 
divergence-free; viz. the vector current must be conserved. Of course the same integrability condition is seen in the original vector formulation of the model, with equation of motion (2.8), which entails conservation of the vector current (dual to the axial vector current).

But let us suppose that we have dynamical information only about the topological variables, and do not know whether the current dual to the axial vector current is conserved. (This is the situation that we shall meet in four dimensions.) Then we must reformulate our theory in such a way that the integrability condition is avoided.

This reformulation in two dimensions proceeds by introducing two Stückelberg fields $p$ and $q$ into $\mathcal{L}_{2}$.

$$
\mathcal{L}_{2}^{\prime}=\frac{1}{2} \mathcal{P}_{2}^{2}+e\left(\mathcal{C}_{2}^{\alpha}+\epsilon^{\alpha \beta} \partial_{\beta} p\right)\left(\mathcal{J}_{\alpha}^{5}+\epsilon_{\alpha \gamma} \partial^{\gamma} q\right)
$$

Upon varying $\mathcal{C}_{2}^{\alpha}$, (3.5) becomes replaced by

$$
-\partial_{\alpha} \mathcal{P}_{2}+e\left(\mathcal{J}_{\alpha}^{5}+\epsilon_{\alpha \gamma} \partial^{\gamma} q\right)=0
$$

Additionally, variation of $p$ and $q$ give, respectively

$$
\begin{aligned}
& \partial_{\alpha} \epsilon^{\alpha \beta} \mathcal{J}_{\beta}^{5}+\partial^{2} q=0 \\
& \partial^{\alpha} \epsilon_{\alpha \beta} \mathcal{C}_{2}^{\beta}+\partial^{2} p=0 .
\end{aligned}
$$

The integrability condition on (3.7) demands that the curl of $\mathcal{J}_{\alpha}^{5}+\epsilon_{\alpha \gamma} \partial^{\gamma} q$ vanish but this is secured by (3.8). This equation determines a non-trivial value for $q$ if the curl of $\mathcal{J}_{\alpha}^{5}$ is nonvanishing, while (3.9) fixes an innocuous value for $p$. Finally we observe that the divergence of (3.7) annihilates the $q$ - dependent term, leaving in the end the previous equation (2.11).

We may understand the role of the Stückelberg fields by reverting to the original vector variables. Then the interaction part of $\mathcal{L}_{2}^{\prime}$ in (3.6) reads

$$
\mathcal{L}_{2 I}^{\prime}=-e\left(\mathcal{J}^{\mu}+\partial^{\mu} q\right)\left(A_{\mu}+\partial_{\mu} p\right)
$$

and (3.8), (3.9) have respective counterparts in

$$
\begin{aligned}
& \partial_{\mu} \mathcal{J}^{\mu}+\partial^{2} q=0, \\
& \partial^{\mu} \mathcal{A}_{\mu}+\partial^{2} p=0 .
\end{aligned}
$$

Eliminating $p$ and $q$ from (3.10) with the help of (3.11), (3.12) leaves

$$
\mathcal{L}_{2 I}^{\prime}=-e \mathcal{J}^{\mu}\left(\delta_{\mu}^{\nu}-\frac{\partial_{\mu} \partial^{\nu}}{\partial^{2}}\right) A_{\nu}
$$


This shows that the Stückelberg fields ensure that the interaction occurs only between transverse components of $\mathcal{J}^{\mu}$ and $A_{\mu}$. For yet another perspective on the role of the Stückelberg fields, note that $-e \int \mathcal{J}^{\mu} A_{\mu}$ is not gauge invariant $\left(A_{\mu} \rightarrow A_{\mu}+\partial_{\mu} \theta\right)$ when $\mathcal{J}^{\mu}$ is not conserved. However, the combination $A_{\mu}+\partial_{\mu} p$ is always gauge invariant because $p$ can transform as $p-\theta$. Finally observe that eliminating the Stückelberg fields in (3.5) with the help of (3.8) and the anomaly equation (2.7) leaves

$$
\partial_{\mu}\left(\mathcal{P}_{2}+\frac{e^{2} / \pi}{\partial^{2}} \mathcal{P}_{2}\right)=0
$$

This is equivalent to (3.5), but carries no integrability condition. Thus we see that the Stückelberg modification overcomes difficulties, which arise when the current dual to the axial vector is not conserved.

\section{4-Dimensional Model with Topological Mass Generation}

For a 4-dimensional generalization of the previous, we adopt the formulation of the 2-dimensional model, presented in Section III in terms of the Chern-Pontryagin density and Chern-Simons current, now promoted to four dimensions, $\mathcal{P}_{4}$ and $\mathcal{C}_{4}^{\alpha}$ respectively, with the latter coupling to an axial vector current $\mathcal{J}_{\alpha}^{5}$ whose divergence is anomalous. The topological entities are constructed from gauge potentials, which we take to be Abelian or non-Abelian; in either case $\mathcal{P}_{4}$ and $\mathcal{C}_{4}^{\alpha}$ remain gauge singlets.

$$
\begin{gathered}
\mathcal{P}_{4} \equiv \frac{1}{2} \epsilon^{\alpha \beta \mu \nu} F_{\alpha \beta}^{a} F_{\mu \nu}^{a}={ }^{*} F^{\mu \nu}{ }^{a} F_{\mu \nu}^{a} \\
F_{\mu \nu}^{a} \equiv \partial_{\mu} A_{\nu}^{a}-\partial_{\nu} A_{\mu}^{a}+f^{a b c} A_{\mu}^{b} A_{\nu}^{c}, \quad{ }^{*} F^{\alpha \beta} \equiv \frac{1}{2} \epsilon^{\alpha \beta \mu \nu} F_{\mu \nu} \\
\mathcal{C}_{4}^{\alpha} \equiv 2 \epsilon^{\alpha \mu \nu \omega}\left(A_{\mu}^{a} \partial_{\nu} A_{\omega}^{a}+\frac{1}{3} f^{a b c} A_{\mu}^{a} A_{\nu}^{b} A_{\omega}^{c}\right) \\
\partial_{\alpha} \mathcal{C}_{4}^{\alpha}=\mathcal{P}_{4}
\end{gathered}
$$

Here $f^{a b c}$ are the structure constants of the appropriate Lie algebra.

Unlike in the 2-dimansional case, the Chern-Simons current is not linear in the gauge vector potential; nevertheless we remain with the potential as the fundamental dynamical variable, and the variation of the Chern-Simons current reads

$$
\delta \mathcal{C}_{4}^{\alpha}=4^{*} F^{\alpha \mu a} \delta A_{\mu}^{a}-2 \epsilon^{\alpha \nu \omega \mu} \partial_{\nu}\left(A_{\omega}^{a} \delta A_{\mu}^{a}\right)
$$


A further difference from the Schwinger model is that there is no reason to suppose that the dual to the 4-dimensional axial vector current is conserved. On the level of 4-dimensional gamma matrices, the duality relation is

$$
\epsilon^{\mu \nu \omega \alpha} \gamma_{\alpha} \gamma^{5}=g^{\mu \nu} \gamma^{\omega}-g^{\mu \omega} \gamma^{\nu}+g^{\nu \omega} \gamma^{\mu}-\gamma^{\mu} \gamma^{\nu} \gamma^{\omega}
$$

It is improbable that fermion dynamics (here unspecified) would leave conserved the current dual to the axial vector current. But this is not an obstacle to our construction, because we can employ the Stückelberg formalism, as explained in the previous Section, to overcome the difficulty.

Thus the Lagrange density that we adopt is

$$
\mathcal{L}_{4}^{\prime}=\frac{1}{2} \mathcal{P}_{4}^{2}+\Lambda^{2}\left(\mathcal{C}_{4}^{\alpha}+\partial_{\beta} p^{\alpha \beta}\right)\left(\mathcal{J}_{\alpha}^{5}+\partial^{\gamma} q_{\alpha \gamma}\right)
$$

The Stückelberg fields $p^{\alpha \beta}$ and $q_{\alpha \gamma}$ are anti symmetric in their indices; $\Lambda^{2}$ carries mass-squared dimension; the axial vector current possesses an anomalous divergence.

$$
\partial^{\alpha} \mathcal{J}_{\alpha}^{5}=-N^{*} F^{\mu \nu a} F_{\mu \nu}^{a}=-N \mathcal{P}_{4}
$$

$N$ is a numerical coupling constant, taken positive.

Variation of the $\mathcal{L}_{4}^{\prime}$ action with respect to $A_{\mu}^{a}$ gives, with the help of (4.4),

$$
\begin{gathered}
\int\left(-\partial_{\alpha} \mathcal{P}_{4}+\Lambda^{2}\left(\mathcal{J}_{\alpha}^{5}+\partial^{\gamma} q_{\alpha \gamma}\right)\right) \delta \mathcal{C}_{4}^{\alpha}= \\
\int\left[4\left(-\partial_{\alpha} \mathcal{P}_{4}+\Lambda^{2}\left(\mathcal{J}_{\alpha}^{5}+\partial^{\gamma} q_{\alpha \gamma}\right)\right)^{*} F^{\alpha \mu a}-2 \epsilon^{\alpha \nu \omega \mu} A_{\nu}^{a} \partial_{\omega}\left(-\partial_{\alpha} \mathcal{P}_{4}+\Lambda^{2}\left(\mathcal{J}_{\alpha}^{5}+\partial^{\gamma} q_{\alpha \gamma}\right)\right)\right] \delta A_{\mu}^{a},
\end{gathered}
$$

so that the equation of motion demands

$$
2\left(-\partial_{\alpha} \mathcal{P}_{4}+\Lambda^{2}\left(\mathcal{J}_{\alpha}^{5}+\partial^{\gamma} q_{\alpha \gamma}\right)\right)^{*} F^{\alpha \mu a}-\epsilon^{\alpha \mu \nu \omega} A_{\nu}^{a} \partial_{\omega} \Lambda^{2}\left(\mathcal{J}_{\alpha}^{5}+\partial^{\gamma} q_{\alpha \gamma}\right)=0 .
$$

Variation of the two Stückelberg fields yields the equations

$$
\begin{aligned}
& \partial_{\alpha}\left(\mathcal{J}_{\beta}^{5}+\partial^{\gamma} q_{\beta \gamma}\right)-\alpha \longleftarrow \beta=0, \\
& \partial^{\alpha}\left(\mathcal{C}_{4}^{\beta}+\partial_{\gamma} p^{\beta \gamma}\right)-\alpha \longleftarrow \rightarrow \beta=0 .
\end{aligned}
$$

The first of these allows setting to zero the second member of (4.9), while in the first member of that equation we may strip away ${ }^{*} F^{\alpha \mu}$ a with the help of the identity

$$
{ }^{*} F^{\alpha \mu} F_{\mu \nu}=-\frac{1}{4} \delta_{\nu}^{\alpha} \mathcal{P}_{4}
$$


Consequently (provided $\mathcal{P}_{4} \neq 0$ ) we are left with

$$
-\partial_{\alpha} \mathcal{P}_{4}+\Lambda^{2}\left(\mathcal{J}_{\alpha}^{5}+\partial^{\gamma} q_{\alpha \gamma}\right)=0
$$

The integrability condition on this equation is satisfied by virtue of (4.10). Taking another divergence of (4.13) annihilates the Stückelberg field because of its anti symmetry, while (4.7) provides the divergence for $\mathcal{J}_{\alpha}^{5}$. Thus we are left with

$$
\partial^{2} \mathcal{P}_{4}+N \Lambda^{2} \mathcal{P}_{4}=0
$$

This shows that the pseudoscalar $\mathcal{P}_{4}$ has acquired the mass, $m^{2}=N \Lambda^{2}$.

By taking the divergence of (4.10), we find from (4.7)

$$
\mathcal{J}_{\beta}^{5}+\partial^{\gamma} q_{\beta \gamma}=-\frac{N}{\partial^{2}} \partial_{\beta} \mathcal{P}_{4}
$$

Inserting this in (4.13) yields

$$
\partial_{\alpha}\left(\mathcal{P}_{4}+\frac{N \Lambda^{2}}{\partial^{2}} \mathcal{P}_{4}\right)=0
$$

which is equivalent to (4.14), but does not entail integrability conditions.

\section{Conclusion}

While the 4-dimensional transposition of the 2-dimensional Schwinger model succeeds in generating a mass for a pseudoscalar, just as in the 2-dimensional case, there are various shortcomings. To these we now call attention.

The principal defect is the absence of dynamics that should produce the anomaly for the axial vector current. In the Schwinger model, the same dynamics and the same degrees of freedom that generate the mass are also responsible for the anomaly (2.7). In the 4-dimensional theory we must posit the anomaly (4.7) separately from the mass generating dynamics. Moreover, our final result is that $\mathcal{P}_{4}$ propagates as a free massive field. Additional dynamics must be specified to describe interactions.

A related question concerns the role in physical theory for our Lagrangian (4.6). Since it involves dimension eight $\left(\mathcal{P}_{4}\right)$ and dimension six $\left(\mathcal{C}_{4}^{\alpha} \mathcal{J}_{\alpha}^{5}\right)$ operators, it should be viewed as an effective Lagrangian. In this connection, observe that the Born-Infeld action and the radiatively induced Euler-Heisenberg action both contain the Abelian $\left({ }^{*} F^{\mu \nu} F_{\mu \nu}\right)^{2}$ quantity in a weak-field expansion [also accompanied by an $\left(F^{\mu \nu} F_{\mu \nu}\right)^{2}$ term].

The kinetic portion of the Lagrangian in the Weyl $\left(A_{0}=0\right)$ gauge involves $\dot{A}_{i} \dot{A}_{j} B^{i} B^{j}$ where $B^{i}$ is the magnetic field. Canonical analysis and quantization with such a kinetic term faces 
difficulties because the metric on $A_{i}$ space, viz. $B^{i} B^{j}$, is singular. But this poses no problem if our Lagrangian is used for phenomenological purposes, with the semi-classical addition of quantum effects through the chiral anomaly.

The $U(1)$ character of our anomalous current and the presence in our theory of the ChernPontryagin quantity suggest that here we are dealing with the problems of the unwanted axial $U(1)$ symmetry and the mass of the $\eta^{\prime}$ meson. Conventionally these issues are resolved by instantons [4]. Here we offer a phenomenological description. We relate the axial vector current to the $\eta^{\prime}$ field,

$$
\mathcal{J}_{\alpha}^{5}=Z \partial_{\alpha} \eta^{\prime} / \Lambda
$$

( $Z$ is a normalization) and add an $\eta^{\prime}$ kinetic term to (4.6).

$$
\mathcal{L}_{\eta^{\prime}}=\frac{1}{2} \mathcal{P}_{4}^{2}+Z \Lambda \mathcal{C}_{4}^{\alpha} \partial_{\alpha} \eta^{\prime}+\frac{1}{2} \partial_{\alpha} \eta^{\prime} \partial^{\alpha} \eta^{\prime}
$$

[We dispense with the Stückelberg fields because the dual of the current in (5.1) is conserved.] Observe that the $\eta^{\prime}$ field enjoys a constant shift symmetry, as befits the quadratic portion of a Goldstone field Lagrangian. The equations that follow from varying $A_{\mu}^{a}$ and $\eta^{\prime}$ respectively, are

$$
\begin{gathered}
\partial_{\alpha}\left(\mathcal{P}_{4}-Z \Lambda \eta^{\prime}\right)^{*} F^{\alpha \mu a}=0 \\
\partial^{2} \eta^{\prime}+Z \Lambda \mathcal{P}_{4}=0
\end{gathered}
$$

Together the two imply

$$
\partial^{2} \mathcal{P}_{4}+Z^{2} \Lambda^{2} \mathcal{P}_{4}=0
$$

As before, a mass is generated.

This may also be seen by rewriting the Lagrangian in (5.2), apart from a total derivative, as

$$
\begin{aligned}
\mathcal{L}_{\eta^{\prime}} & =\frac{1}{2} \mathcal{P}_{4}^{2}-Z \Lambda \mathcal{P}_{4} \eta^{\prime}+\frac{1}{2} \partial_{\alpha} \eta^{\prime} \partial^{\alpha} \eta^{\prime} \\
& =\frac{1}{2}\left(\mathcal{P}_{4}-Z \Lambda \eta^{\prime}\right)^{2}+\frac{1}{2} \partial_{\alpha} \eta^{\prime} \partial^{\alpha} \eta^{\prime}-\frac{1}{2} Z^{2} \Lambda^{2} \eta^{\prime 2} .
\end{aligned}
$$

With $Z \Lambda \eta^{\prime}$ absorbed by $\mathcal{P}_{4}$, we see that $\eta^{\prime}$ decouples, but carries a mass.

In the case of 4-dimensional QCD with massless quark flavor(s), equation (5.5) can be obtained without any assumptions about the dependence of the effective Lagrangian on the $\eta^{\prime}$ meson. We only need to assume that the effective Lagrangian contains the first $\mathcal{P}_{4}^{2}$ term in (5.6). The analog of the second term is automatically generated from the anomaly diagram (Fig. 2) that correlates $\mathcal{C}_{4}^{\alpha}$ and $\mathcal{J}_{\alpha}^{5}$. 


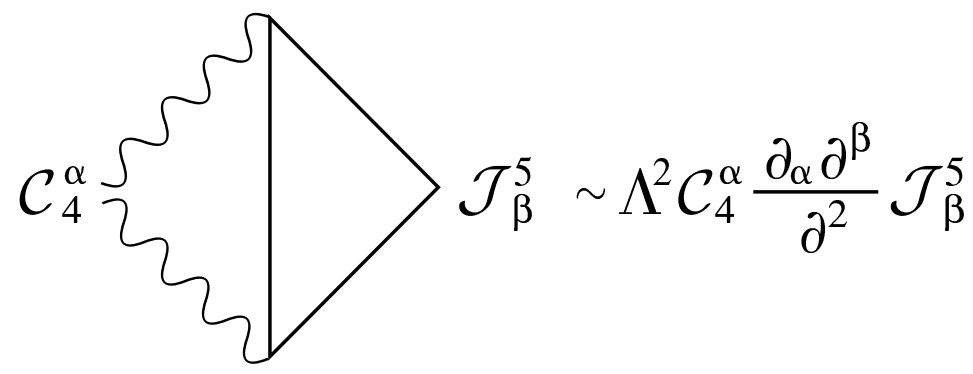

Figure 2: Anomaly diagram that correlates $\mathcal{C}_{4}^{\alpha}$ and $\mathcal{J}_{\beta}^{5}$

The diagram generates the following operator

$$
\Lambda^{2} \mathcal{C}_{4}^{\alpha} \frac{\partial_{\alpha} \partial^{\beta}}{\partial^{2}} \mathcal{J}_{\beta}^{5}
$$

where $\Lambda^{2}$ arises as a momentum cut off. This expression is also what one obtains from (4.6) after eliminating the Stückelberg fields $p^{\alpha \beta}$ and $q_{\alpha \gamma}$ through their equations of motion (4.10), (4.11). Thus massless quark dynamics due to the anomaly substitute the effect of the Stückelberg fields. Variation with respect to $A_{\mu}^{a}$ yields the analog of equation (4.13).

$$
-\partial_{\alpha} \mathcal{P}_{4}+\Lambda^{2} \frac{\partial_{\alpha}}{\partial^{2}} \partial^{\beta} \mathcal{J}_{\beta}^{5}=0
$$

Using the anomalous divergence relation (4.7), we arrive to the equation (4.16), which is equivalent to (5.5). Because $\mathcal{P}_{4}$ acquires a mass, its expectation value in the QCD vacuum must vanish. This explains why QCD solves both $U(1)$ and the strong CP problems in the zero quark mass limit [5].

Similar effects should be present in all even dimensions, but the singularity structure and the required dimensional parameter (analog of the 2- and 4-dimensional $e$ and $\Lambda$ ) will change.

In conclusion we observe that although both the 2- and 4-dimensional models are formulated in terms of topological entities $\left(\mathcal{P}, \mathcal{C}^{\alpha}\right)$, they are not topological theories. Examining $(3.6),(4.6)$ we see that the Chern-Simons/axial vector interaction term $\left(\mathcal{C}^{\alpha} \mathcal{J}_{\alpha}^{5}\right)$ is a geometric scalar density and can be integrated over a manifold in a diffeomrphism invariant way, without introducing a metric tensor. However, for the kinetic term $\left(\mathcal{P}^{2}\right)$ to be a scalar density it must be divided by $\sqrt{g}$. (In this discussion we ignore the Stückelberg terms.) Without this metric factor the theory is not invariant against all diffeomorphisms, but only against the volume preserving ones with unit Jacobian.

\section{Acknowledgments}

We thank G. Gabadaze and R.L. Jaffe for useful discussions. The work of G. D. is supported in part by David and Lucile Packard Foundation Fellowship for Science and Engineering, and 
by NSF grant PHY-0245068; that of R.J. and S.Y. Pi by DOE grants DE-FCO2-94ER-40818 and DE-FG02-91ER-40676, respectively.

\section{References}

[1] J. Schwinger, Phys. Rev. 125, 397 (1962); Phys. Rev. 128, 2425 (1962).

[2] The relevant topological structures are discussed by e.g. R. Jackiw in "Fifty Years of Yang-Mills Theory", G. 't Hooft, ed. (World Scientific, Singapore, 2005).

[3] The anomaly-based approach in the Schwinger model is explained in the Introduction to "Dynamical Gauge Symmetry Breaking", E. Farhi and R. Jackiw, eds. (World Scientific, Singapore, 1982).

[4] G. 't Hooft, Phys. Rept. 142, 357 (1986).

[5] G. Dvali, [arxiv: hep-th/0507215. 